\title{
Modified Barium Swallow
}

National Cancer Institute

\section{Source}

National Cancer Institute. Modified Barium Swallow. NCI Thesaurus. Code C125909.

A fluoroscopic procedure that examines a person's swallowing pattern. Liquid and solid food that contains barium sulfate is digested to determine if it is entering a person's larynx and trachea. 